\title{
Measurement of lumbar muscle glucose utilization rate can be as useful in estimating skeletal muscle insulin resistance as that of thigh muscle*
}

\author{
Ikuo Yokoyama ${ }^{1,2 \#}$, Toshiyuki Moritan ${ }^{3}$, Yusuke Inoue ${ }^{4}$ \\ ${ }^{1}$ Department of Cardiovascular Medicine, Clinical Research Center, Sanno Medical Center Hospital, International University of \\ Health and Welfare, Tokyo, Japan \\ ${ }^{2}$ Department of Cardiovascular Medicine, Clinical Research Center, Sanno Hospital, International University of Health and Welfare, \\ Tokyo, Japan \\ ${ }^{3}$ Department of Clinical Engineering, Faculty of Medical Engineering, Suzuka University of Medical Science, Suzuka, Japan \\ ${ }^{4}$ Department of Radiology, Graduate School of Medicine, Kitasato University, Sagamihara, Japan \\ Email: \#yokochan-tky@umin.ac.jp
}

Received 2 November 2012; revised 1 December 2012; accepted 7 December 2012

\begin{abstract}
Background: Skeletal muscle glucose utilization (SMGU) can be accessed by positron emission tomography (PET) and 18F-FDG to characterize insulin resistance. The quantity of skeletal muscle in the lumbar is sufficient to indicate that SMGU in the lumbar (SMGUlumbar) can be measured with 18F-FDG PET of the chest instead of obtaining thigh muscle SMGU (SMGU-thigh). This would reduce PET scan time to avoid thigh muscle PET scan. This study was aimed to compare SMGU-lumbar and thigh muscle SMGU under insulin clamping to identify the validity of measurements of SMGU in the lumbar for studies of insulin resistance. Methods: Thirty-three patients underwent sequential dynamic 18F-FDG PET of both the thoracic (37 $\mathrm{min})$ and thigh region $(22 \mathrm{~min})$ during hyperinsulinemic euglycemic insulin clamping. Both SMGU-lumbar and SMGU-thigh were calculated by Patlak graphical analysis. Whole body insulin resistance was assessed by a whole body glucose disposal rate during hyperinsulinemic euglycemic insulin clamping. Input function was obtained from the time activity curve of the descending aorta and venous blood sampling as previously validated. Results: SMGU-thigh $(0.0506 \pm 0.0334 \boldsymbol{\mu m o l} / \mathrm{min} / \mathrm{g})$ was comparable to SMGU-lumbar $(0.0497 \pm 0.0255 \mu \mathrm{mol} / \mathrm{min} / \mathrm{g})$. The Bland-Altman method of difference plot analysis showed a significant correlationship between SMGUthigh and SMGU-lumbar $(r=0.506, p=0.0028)$. There were seen very good significant correlationship between whole body glucose utilization rate in both thigh $(r=0.737, p=0.0001)$ and lumbar $(r=0.772, p$
\end{abstract}

\footnotetext{
${ }^{*}$ There are non-financial competing interests with this article.

${ }^{\#}$ Corresponding author.
}

$=0.0001)$. Conclusion: These results support the validity of measuring SMGU-lumbar to estimate insulin resistance during $P E T$ imaging of the chest.

Keywords: ${ }^{18}$ F-FDG; PET; Skeletal Muscle; Glucose; Insulin Resistance; Metabolic Syndrome; Type II Diabetes; Hypertension

\section{INTRODUCTION}

Insulin resistance, a decreased glucose utilization response to the stimulatory effect of insulin, is accepted to be of critical importance in various metabolic abnormalities leading to atherosclerosis such as essential hypertension, diabetes mellitus, hypertriglyceridemia, and/or a combination of these diseases, such as in metabolic syndrome, etc. Positron emission tomography (PET) allows the estimation of the glucose utilization rate in vivo using $\left[{ }^{18} \mathrm{~F}\right]$-2-fluoro-2-deoxy-D-glucose $\left({ }^{18} \mathrm{~F}\right.$-FDG) as a tracer and can provide valuable insights into insulin resistance in the clinical setting. A major advantage of the PET technique resides in its quantitative ability to assess an organ-specific glucose utilization. Skeletal muscle glucose utilization rate (SMGU) is thought to be the most essential to the whole body insulin resistance, and measurements of SMGU under insulin stimulation with PET have been reported [1]. Glucose is an essential substrate as a predominant source of energy for skeletal muscle to maintain normal skeletal muscle function, and insulin resistance plays an important role in the development of coronary artery disease (CAD) [2-4]. An existence of insulin resistance in the heart has been reported [5-9] but it is not always pararel to the skeletal muscle and/or whole body insulin resistance [10-14]. Therefore, simultaneous PET measurement of myocardial glucose utilize- 
tion rate (MGU) and SMGU has been done [5-15] and results in such studies might lead to characterize insulin resistance in various diseases and increase our knowledge of therapies for insulin resistance.

SMGU can be calculated by Patlak graphical analysis [16], which requires the temporal profile of tissue activity on PET images as the output function and the temporal profile of arterial plasma activity as the input function. To measure glucose utilization in the chest using PET scan, the input function can be given without arterial blood sampling using the time-activity curve of the left ventricle (LV) [17] or descending aorta [18]. Sequential measurement of both MGU and thigh muscle SMGU (SMGU (thigh)) following an injection of ${ }^{18} \mathrm{~F}-\mathrm{FDG}$ has been used. In the sequential method, the chest PET scan is commonly performed immediately after tracer injection, followed by data collection in the thigh region, with tissue activity only during the late phase used to calculate SMGU (thigh). The input function can be determined from chest PET images for the early phase and from arterialized venous blood samples during the late phase just at the time of the thigh muscle PET scan [10-13,19]. A non-invasive method to measure SMGU (thigh) using a sequential PET scan of the chest and thigh region that does not require arterial blood sampling and an early to mid dynamic PET scan of the thigh region has been reported [20]. Since there exists sufficient amount of skeletal muscle in the lumbar region, measurements of SMGU in the lumbar (SMGU (lumbar)) can be obtained from an ${ }^{18}$ F-FDG PET scan of the chest using previously validated non-invasive method [20]. By using that method [20], MGU, SMGU (lumbar) and SMGU (thigh) can be calculated with one sequential PET scan with only 3 - 5 times of venous blood sampling leading to avoiding arterial blood sampling. Moreover, if there were no difference in SMGU value between the lumbar and thigh region, thigh muscle PET imaging can be avoided leading to decrease the PET data sampling time to study heart and skeletal muscle insulin resistance. If there were differences in the SMGU value and/or the relationship between SMGU and the whole body glucose utilization rate (WBGU) between the lumbar and thigh, measurement of SMGU in both regions would provide additional information to increase our knowledge of several diseases with insulin resistance. Since PET data acquisition time with ${ }^{18} \mathrm{~F}$-FDG under insulin clamping might be reduced as shorter as possible because of too long preparation time more than 2 hours to obtain steady state condition of glucose utilization under insulin claming before the start of ${ }^{18}$ F-FDG PET scan. Therefore, when considering simultaneous measurement of MGU and SMGU, SMGU (lumbar) is preferred because data sampling time can be decreased to estimate SMGU. Thus, the aim of this study is to elucidate both differences and similarities in the
SMGU value and its relationships to WBGU between the thigh and lumbar and demonstrate whether SMGU (lumbar) could be used in studies of skeletal muscle insulin resistance leading to reduce PET data sampling time.

\section{MATERIALS AND METHODS}

\subsection{Study Subjects}

Sequential dynamic ${ }^{18}$ F-FDG PET scans of the chest and thigh regions were performed in 34 patients. Patient data obtained for a previous study that had different objectives from the present report were used [20,21]. One patient moved considerably during the PET scan, making evaluation of SMGU unreliable, and thus data from this patient were excluded from analysis. Consequently, data on 33 patients ( 1 woman, 32 men; age range 27 - 77 years, mean age $57.5 \pm 9.3$ years) were analyzed. Thirtytwo patients had type II diabetes, and one had only essential hypertension. Average of HbA1c (\%) was $7.2 \% \pm$ $1.3 \%$. Patients with Type II diabetes or essential hypertension who had been admitted to our hospital or local health service centers were enrolled in this study. No other specific inclusion criteria were employed. The investigative nature of the study was fully explained to each patient before informed consent was obtained. The local ethics committee approved the study protocol.

\subsection{Imaging Procedures}

Dynamic PET scan with ${ }^{18} \mathrm{~F}-\mathrm{FDG}$ was performed during hyperinsulinemic euglycemic clamping. ${ }^{18} \mathrm{~F}$ was synthesized using the Cypris model 370 cyclotron (Sumitomo JYUKI Industries, Ltd., Tokyo, Japan), and ${ }^{18} \mathrm{~F}-\mathrm{FDG}$ was synthesized with an automated system based on the method reported by Ehrenkaufer et al. [22]. Radiochemical purity was more than $95 \%$. A Headtome IV PET scanner (Shimadzu Corp., Kyoto, Japan) was used for PET scans. Transmission data were acquired before the administration of ${ }^{18} \mathrm{~F}$-FDG to correct for photon attenuation. Two venous catheters were inserted: one in a superficial forearm vein for the infusion of glucose and insulin and the injection of ${ }^{18} \mathrm{~F}-\mathrm{FDG}$, and one in a superficial vein of the contralateral forearm for venous blood sampling. Prior to the PET scan, a primed-constant insulin infusion 4 times the final constant rate $(1 \mathrm{mlU} / \mathrm{min} / \mathrm{kg})$ for the first 4 min was started after which the insulin infusion was 2 times the final constant infusion rate for an additional $4 \mathrm{~min}$. The exogenous glucose infusion using $20 \%$ glucose solution was started when the insulin infusion was at the constant rate. Plasma glucose concentration was measured at baseline and then every 5 min during insulin clamping. The glucose infusion rate was adjusted according to the change in plasma concentration and targeted at about $5.6 \mu \mathrm{mol} / \mathrm{L}$. Under near steady- 
state conditions, the exogenous glucose infusion rate equals the total amount of glucose metabolized by all tissues. We determined the WBGU as follows: WBGU = the exogenous glucose infusion rate $(\mathrm{mg} / \mathrm{min}) /$ body weight $(\mathrm{kg})$.

When the plasma glucose concentration became constant, ${ }^{18}$ F-FDG (185 - $\left.370 \mathrm{MBq}\right)$ was injected intravenously over $60 \mathrm{~s}$.

Sequential PET scanning of the thoracic and thigh regions was performed. Immediately after tracer injection, dynamic data on the thoracic region, including the heart in the field of view, were acquired for $37 \mathrm{~min}$ (10 $\mathrm{s} /$ frame $\times 9,30 \mathrm{~s} /$ frame $\times 3,120 \mathrm{~s} /$ frame $\times 2,300 \mathrm{~s} /$ frame $\times 4$, and $600 \mathrm{~s} /$ frame $\times 1$ ), followed by the collection of dynamic data of the mid-thigh region for $22 \mathrm{~min}(120$ $\mathrm{s}$ /frame $\times 1$ and $300 \mathrm{~s} /$ frame $\times 4$ ). A venous blood sample was obtained at the midpoint of each frame for the last 7 frames of thoracic imaging and for all of the frames of thigh imaging.

\subsection{Data Analysis}

PET data were corrected for dead time, decay, and measured photon attenuation, and transaxial images were reconstructed. Fifteen circular regions of interest (ROIs) of $5 \mathrm{~mm}$ in diameter were placed within the lumbar muscle and thigh muscles of each side. Orientation of lumbar muscle was made in relation to specific vertebrae since lumbar muscle is just behind the vertebral column. We also defined lumbar muscle ${ }^{18}$ F-FDG activity by changing the color scale level for the thoracic region to view lumbar muscle adequately and to negate the deleterious influence of cardiac muscle activity. An example of ROI placement for back muscle is shown in Figure 1. Tissue activity was determined from the mean counts for the ROIs. Plasma and tissue time-activity curves were analyzed by the graphical method described by Patlak et al. [17] to determine the fractional rate of tracer uptake and phosphorylation $K_{i} \cdot K_{i}$ is equal to $k_{1} \times k_{3} /\left(k_{2}+k_{3}\right)$, where $k_{1}$ is the transfer coefficient from intravascular space into the tissue, $k_{2}$ is the initial clearance and efflux

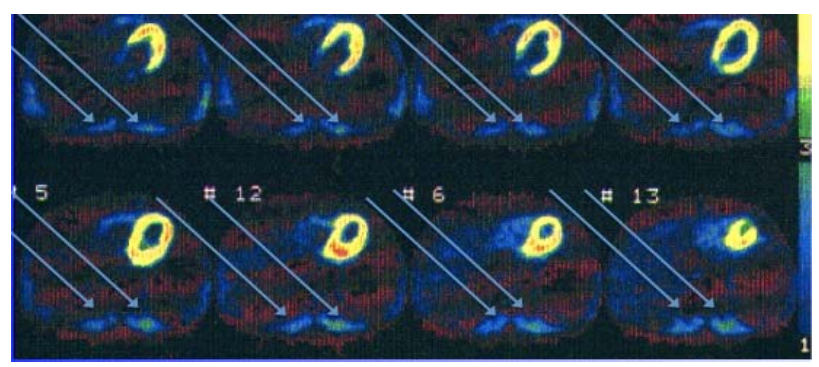

Figure 1. A typical example of lumbar muscle static ${ }^{18} \mathrm{~F}-\mathrm{FDG}$ uptake positron emission tomography (PET) image (arrow headed). Regions of interests (ROIs) were placed reffering the static lumbar muscle ${ }^{18}$ F-FDG uptake PET images. coefficient, and $k_{3}$ is the phosphorylation rate constant. The dephosphorylation rate constant, $k_{4}$, is assumed to be zero with the Patlak method. SMGU was calculated by substituting $K_{i}$ in the equation $\mathrm{SMGU}=K_{i} \times \mathrm{Gl}_{\mathrm{p}} / \mathrm{LC}$, where $\mathrm{Gl}_{\mathrm{p}}$ is the mean of the venous plasma glucose level during imaging and $\mathrm{LC}$ is the lumped constant. LC strands for differences in the transport and phosphorylation of ${ }^{18} \mathrm{~F}-\mathrm{FDG}$ and glucose, and was estimated at 1.2 $[23,24]$. To calculate SMGU, we used an Ultra 30 highspeed image processing system (Sun Microsystems Japan, Tokyo, Japan) with Dr. View software (Asahi Kasei Information System Co., Ltd., Tokyo, Japan).

In the Patlak graphical analysis, the slope of the linear portion of the plots, which is equal to

$K_{i}\left(k_{1} \times k_{3} /\left(k_{2}+k_{3}\right)\right)$ of the ${ }^{18}$ F-FDG three-compartment tracer kinetic model, is calculated by linear regression. SMGU (lumbar) was determined based on linear regression of the last 7 points during the chest PET scan based on the method by Ohtake et al. [18]. The time activity curve of the descending aorta corrected by venous blood sampling was used as the input function according to the method by Ohtake et al. [18]. The time-activity curve for the descending aorta was generated and corrected using venous blood samples. Input function during the thigh imaging was assessed using venous blood samples. Thigh muscle SMGU with the estimated input function was calculated using the combination of the corrected aorta activity during the chest PET scan and venous plasma activity during the thigh PET scan as described previously [20].

Measurements of whole body insulin resistance were made by obtaining the glucose disposal rate during hyperinsulinemic euglycemic clamping $(\mu \mathrm{mol} / \mathrm{min} / \mathrm{kg})$ just at the time of the PET scan using a previously reported method [25].

\subsection{Statistical Analysis}

Data are expressed as means \pm standard deviation (SD). The Bland-Altman method of difference plot analysis was used to compare the two methods. A p value of less than 0.05 was considered statistically significant.

\section{RESULTS}

SMGU (thigh) $(0.0506 \pm 0.0334 \mu \mathrm{mol} / \mathrm{min} / \mathrm{g})$ was comparable with SMGU (lumbar) $(0.0497 \pm 0.0255 \mu \mathrm{mol} /-$ $\mathrm{min} / \mathrm{g}$ ). The ratio of SMGU (thigh) to SMGU (lumbar) was $0.992 \pm 0.322$. There was a significant positive relationship between the SMGU (thigh) and SMGU (lumbar) (Figure 2).

The Bland-Altman method of difference plot analysis showed a significant correlation between SMGU (thigh) and SMGU (lumbar) $(\mathrm{r}=0.506, \mathrm{p}=0.0028)$ (Figure 3). 
SMGU (thigh)-SMGU(lumber)

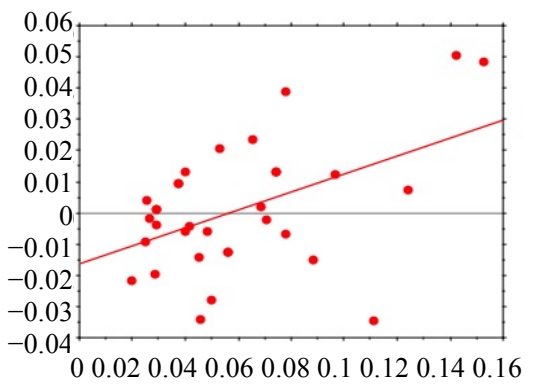

$(\mathrm{SMGU}$ (thigh) $+\mathrm{SMGU}($ back $)) / 2$

Figure 2. Plots of skeletal muscle glucose utilization rate $(\mathrm{SMGU}[\mu \mathrm{mol} / \mathrm{min} / \mathrm{g}])$ (thigh) and SMGU $([\mu \mathrm{mol} / \mathrm{min} / \mathrm{g}])$ (lumbar). There was a significant positive relationship between the two.

WBGU of all study subjects was $34.5 \pm 21.2 \mu \mathrm{mol} /$ $\mathrm{min} / \mathrm{kg}$. A significant positive relationship was found between SMGU thigh and WBGU $(r=0.737, p=0.0001)$ (Figure 4). There was also a significant positive relationship between SMGU (lumbar) and WBGU $(r=0.772$, $\mathrm{p}=0.0001)$ (Figure 5).

\section{DISCUSSION}

In this study, we made sequential ${ }^{18}$ F-FDG PET scans of the chest and thigh regions without arterial blood sampling and calculated both lumbar muscle and thigh muscle SMGU to validate a method to quantify SMGU (lumbar). Nearly the similar good agreement between SMGU (thigh) and SMGU (lumbar) and that between SMGU (lumbar) and WBGU shows that PET imaging of SMGU (lumbar) can be used in evaluating skeletal muscle insulin resistance nearly the same accuracy as that of thigh. In addition, PET imaging of SMGU (lumbar) could decrease data sampling time for the study of heart and skeletal muscle insulin resistance. SMGU (lumbar) can be estimated with only venous blood sampling starting at the time of the ${ }^{18} \mathrm{~F}$-FDG injection and during the dynamic chest PET scan. One of the principal assumptions in applying Patlak graphical analysis to estimate the muscle glucose utilization is that the dephosphorylation of ${ }^{18}$ F-FDG-6-phosphate is negligible in the target tissue. Calculation using tissue ${ }^{18} \mathrm{~F}$-FDG activity obtained at the early to late-mid phase of a PET scan appears to negate the negative influence of dephosphorylation of ${ }^{18}$ F-FDG6-phosphate, which increases during the late phase after ${ }^{18} \mathrm{~F}-\mathrm{FDG}$ injection. That is one of the advantages of the dynamic PET scan using ${ }^{18}$ F-FDG. The extremely low activity of dephosphorylation in skeletal muscle [25] can be an important factor in producing a good agreement between SMGU (lumbar) and SMGU (thigh).
SMGU (thigh)-SMGU(lumber)

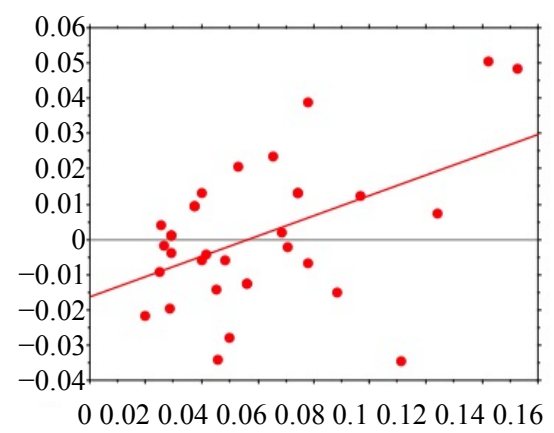

(SMGU (thigh)+SMGU(back))/2

Figure 3. The bland-Altman method of difference plot analysis between skeletal muscle glucose utilization rate (SMGU $[\mu \mathrm{mol} / \mathrm{min} / \mathrm{g}]$ ) (thigh) and SMGU $[\mu \mathrm{mol} / \mathrm{min} / \mathrm{g}])$ (lumbar) showed a significant positive relationship between SMGU thigh and SMGU back $=$ lumbar, $\mathrm{r}=0.868, \mathrm{p}=0.0001$.

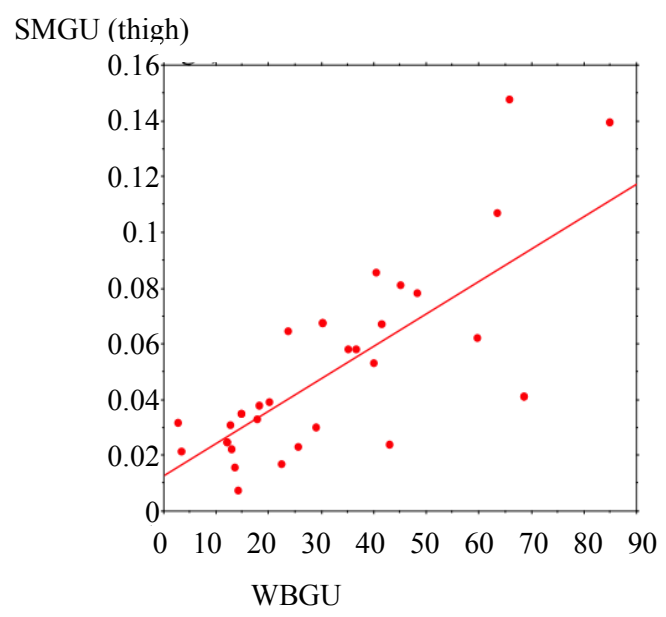

Figure 4. Plots of skeletal muscle glucose utilization rate $(\mathrm{SMGU}[(\mu \mathrm{mol} / \mathrm{min} / \mathrm{g})])($ thigh$)$ and whole body glucose utilization rate (WBGU $[\mu \mathrm{mol} / \mathrm{min} / \mathrm{kg}]$ ). A significant positive relationship was found between SMGU thigh and WBGU $(r=0.737, \mathrm{p}=$ $0.0001)$.

The validity of shortening the PET data sampling time is convenient for both the patients and the PET laboratory. In addition, reducing the total PET data sampling time has merit in estimating the effects of certain drugs such as insulin sensitizers on the heart and skeletal muscle insulin resistance because PET should be done more than two times for the same patients. Several reports have cited an existence of myocardial perfusion abnormalities in subjects with angiographically normal coronary arteries who are at high risk for CAD [27,28] or who have no evidence of CAD $[25,29]$. Reversal effects of certain medicines to improve such myocardial perfu- 


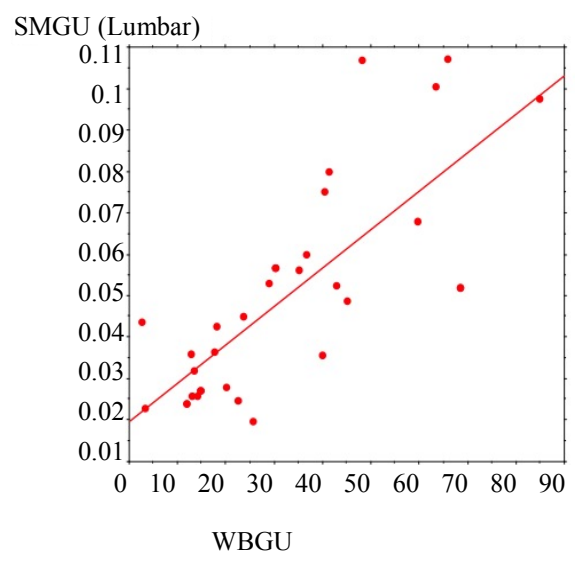

Figure 5. Plots of skeletal muscle glucose utilization rate (SMGU $[\mu \mathrm{mol} / \mathrm{min} / \mathrm{g}]$ ) (lumbar) and whole body glucose utilization rate (WBGU $[\mu \mathrm{mol} / \mathrm{min} / \mathrm{kg}]$ ). There was also a significant positive relationship between SMGU (lumbar) and WBGU $(\mathrm{r}=0.772, \mathrm{p}=0.0001)$.

sion abnormalities have also been reported [30,31]. Since most diseases presenting a risk for CAD such as essential hypertension, type-II diabetes hypertriglyceridemia and abdominal obesity can usually be associated with insulin resistance, it is better to shorten the PET data aquisition time to estimate effects of certain therapies on both the myocardial perfusion abnormalities and heart and skeletal muscle insulin resistance. To achieve such an aim, our method enables measurement of MGU and SMGU (lumbar) simultaneously, while SMGU (thigh) and MGU cannot be measured simultaneously. Measuring both MGU and SMGU and myocardial perfusion abnormalities in a specific subject may aid in evaluating the relation between metabolic abnormalities such as insulin resistance in the heart and skeletal muscle and certain heart diseases. Measurement of SMGU (lumbar) allows for an easier investigation in a much shorter data acquisition period compared with a PET study that includes a heart and thigh muscle ${ }^{18}$ F-FDG PET scan and a myocardial perfusion study. PET scan of the chest can visualize both the myocardium and skeletal muscle in the lumbar, and skeletal muscle in the arm also can be included in the field of view if the patient's arm is at his or her side. MGU and SMGU in the arm [5] may be simultaneously assessed; however, the volume of skeletal muscles in the arm is small, especially in lean patients, which may cause difficulty in placing ROIs and cause underestimation of SMGU due to the partial volume effect. In addition, high activity in the myocardium may interfere with an accurate estimation of SMGU of arm muscle due to star artifacts. Whereas in the present study, apparently obvious ${ }^{18}$ F-FDG uptake was seen in the lumbar muscle associated with high myocardial ${ }^{18} \mathrm{~F}-\mathrm{FDG}$ uptake and no obvious artifact, which exist at lower part of human body than that of heart. Moreover, lumbar muscle has an adequate amount of skeletal muscle to negate partial volume effect. Therefore, such star artifacts could be negated in the measurement of SMGU (lumbar). The thigh region contains a large amount of skeletal muscle and no other structures with high ${ }^{18} \mathrm{~F}$ FDG uptake, and has been thought to be suitable for estimating SMGU. In the present study, individual variability among patients was found in the SMGU value between the lumbar and the thigh. This may be caused by movement of thigh muscle due to long data sampling time (chest PET scan time plus thigh muscle PET scan time), a difference in daily physical activities (lack of exercise, difference in type of job and/or exercise, etc.) and a difference in function between lumbar muscle, which is mainly used to maintain body structure and posture and to breathe, and thigh muscle, which is used to walk and to stand. The much wider range in SMGU values for the thigh than in SMGU values for the lumbar could imply that there are differences in the essential nature of skeletal muscle glucose handling between the two. Moreover, when the SMGU (thigh) value was approximately more than $0.12 \mu \mathrm{mol} / \mathrm{min} / \mathrm{g}$, the difference in the SMGU value between the lumbar and the thigh seemed to be increased. In addition, the range of SMGU (thigh) value was much wider than that in SMGU (lumbar) in spite of the significant positive relationship between the SMGU (thigh) and SMGU (lumbar). The reason for such a discrepancy is speculative. A difference in the timing of PET data sampling between SMGU (lumbar-early to mid phase) and SMGU (thigh - late phase) could be related to such differences. That is one of the limitations of this study. However, the quite excellent agreement in SMGU values between lumbar and thigh muscle in our present study indicated that measurement of SMGU (lumbar) could provide accuracy similar to that of SMGU (thigh) in an absolute estimation of the SMGU value. Moreover, the good relationship between SMGU and WBGU in lumbar muscle was observed to be similar to that between thigh muscle SMGU and WBGU. Therefore, chest PET imaging using the lumbar muscle can offer reasonable estimates of SMGU and skeletal muscle insulin resistance. Since skeletal muscle insulin resistance is a predominant parameter for the whole body insulin resistance, our results might contribute to the study of certain diseases associated with insulin resistance. However, another factors contribute to approximately $20 \%$ of total whole body insulin resistance, so both SMGU, whole body insulin resistance and MGU should be measured simultaneously for the much more accurate understanding of insulin resistance of certain diseases.

Chest PET imaging yields a time-activity curve that represents temporal changes in blood activity, and the input function during thoracic imaging can be deter- 
mined by venous blood sampling alone [17,18]. Although venous plasma activity underestimates peak arterial activity [32], venous blood sampling could suffice for the estimation of the input function during the late phase. Elimination of the need for frequent arterial sampling is a definite convenience. Even without the need for MGU measurement, chest PET imaging provides an assessment of the input function for glucose metabolism in thoracic region and/or SMGU (thigh) with only venous blood sampling instead of arterial blood sampling [20].

The present study aimed to investigate the validity of the methodology for PET measurement of SMGU (lumbar). The results reported here support future investigation of heart and skeletal muscle insulin resistance through methods that are relatively easy with decreased sampling time. Our results also support the avoidance of thigh imaging and the validity of omission of arterial blood sampling in assessing SMGU (lumbar) during hyperinsulinemic euglycemic clamping using the input function derived from the time activity curve of the descending aorta corrected by venous blood sampling [18].

Full compartment model analysis could provide rate constants for the ${ }^{18}$ F-FDG tracer kinetic model leading to obtaining individual quantitative values for the transfer from the intravascular space to tissue $\left(\mathrm{k}_{1}\right)$, the efflux coefficient $\left(\mathrm{k}_{2}\right)$, the phosphorylation rate $\left(\mathrm{k}_{3}\right)$ and eventually the phosphatase activity $\left(\mathrm{k}_{4}\right)$. These analyses would clarify differences and similarities in skeletal muscle glucose utilization between thigh and lumbar muscle under insulin clamping. Because the first aim of this study was to clarify whether SMGU (lumbar) could be estimated similar to the SMGU (thigh), full compartment model analysis (three or more compartment model) was not done. To achieve such an aim as accurately as possible, a simultaneous ${ }^{18}$ F-FDG PET scan for both the lumbar and thigh muscles might be required with Dual PET to obtain full dynamic PET data, which can estimate tissue metabolism, blood flow, etc. simultaneously in two separate portions of the human body [33] and has been used in human heart and brain studies [34-36]. Further investigations would be warranted on this methodology for this purpose.

The influence of the Lumped Constant on the quantitative results in patients with type II diabetes may be one problem in the estimation of SMGU with ${ }^{18} \mathrm{~F}$-FDG PET. Although such an issue is important in the estimation of SMGU, because we compared individual differences in SMGU between thigh and lumbar muscle in the same study subjects, such a problem can be negated.

\section{CONCLUSION}

SMGU (lumbar) could be estimated only by chest PET imaging with accuracy similar to that of thigh muscle SMGU. The results of this study support the validity of simultaneous measurements of MGU and SMGU with only a chest PET scan using ${ }^{18}$ F-FDG and venous blood sampling to estimate insulin resistance syndrome.

\section{AUTHORS' CONTRIBUTIONS}

Dr. Yokoyama made the study design of this study and recruited study subjects. Drs. Yokoyama, Inoue, Moritan made PET data sampling and data analysis. Drs. Inoue, Moritan gave appropriate discussion and advises in making this manuscript.

\section{ACKNOWLEDGEMENTS}

We thank Mr. Tohru Inoue for his technical support in preparing ${ }^{18}$ F-FDG and Dr. Katsunori Yonekura for his kind cooperation.

\section{REFERENCES}

[1] Kelley, D.E., Price, J.C. and Cobelli, C. (2001) Assessing skeletal muscle glucose metabolism with positron emission tomography. IUBMB Life, 52, 279-284. doi:10.1080/152165401317291129

[2] Reaven, G.M. (1988) Banting lecture 1988. Role of insulin resistance in human disease. Diabetes, 37, 1595-1607. doi:10.2337/diabetes.37.12.1595

[3] DeFronzo, R.A. (1992) Insulin resistance, hyperinsulinemia, and coronary artery disease: A complex metabolic web. Journal of Cardiovascular Pharmacology, 20, S1S16. doi:10.1097/00005344-199200111-00002

[4] Després, J.P., Lamarche, B., Mauriège, P., et al. (1996) Hyperinsulinemia as an independent risk factor for ischemic heart disease. The New England Journal of Medicine, 334, 952-957. doi:10.1056/NEJM199604113341504

[5] Voipio-Pulkki, L.M., Nuutila, P., Knuuti, M.J., et al. (1993) Heart and skeletal muscle glucose disposal in type 2 diabetic patients as determined by positron emission tomography. Journal of Nuclear Medicine, 34, 2064-2067.

[6] Yokoyama, I., Ohtake, T., Momomura, S., et al. (1999) Insulin action on heart and skeletal muscle FDG uptake in patients with hypertriglyceridemia. Journal of Nuclear Medicine, 40, 1116-1121.

[7] Peltoniemi, P., Lönnroth, P., Laine, H., et al. (1996) Cardiac and skeletal muscle insulin resistance in patients with coronary heart disease. A study with positron emission tomography. The Journal of Clinical Investigation, 98, 2094-2099. doi:10.1172/JCI119015

[8] Yokoyama, I., Yonekura, K., Ohtake, T., et al. (2000) Role of insulin resistance in heart and skeletal muscle F-18 fluorodeoxyglucose uptake in patients with non-insulin-dependent diabetes mellitus. Journal of Nuclear Cardiology, 7, 242-248. doi:10.1016/S1071-3581(00)70013-4

[9] Iozzo, P., Chareonthaitawee, P., Dutka, D., Betteridge, D.J., Ferrannini, E. and Camici, P.G. (2002) Independent association of type 2 diabetes and coronary artery disease 
with myocardial insulin resistance. Diabetes, 51, 30203024. doi: $10.2337 /$ diabetes.51.10.3020

[10] Nuutila, P., Knuuti, J., Ruotsalainen, U., et al. (1993) Insulin resistance is localized to skeletal but not heart muscle in type 1 diabetes. American Journal of Physiology, 264, E756-E762.

[11] Nuutila, P., Mäki, M., Laine, H., et al. (1995) Insulin action on heart and skeletal muscle glucose uptake in essential hypertension. The Journal of Clinical Investigation, 96, 1003-1009. doi:10.1172/JCI118085

[12] Yokoyama, I., Ohtake, T., Momomura, S., et al. (1998) Organ-specific insulin resistance in patients with noninsulin-dependent diabetes mellitus and hypertension. Journal of Nuclear Medicine, 39, 884-889.

[13] Utriainen, T., Takala, T., Luotolahti, M., et al. (1998) Insulin resistance characterizes glucose uptake in skeletal muscle but not in the heart in NIDDM. Diabetologia, 41, 555-559. doi:10.1007/s001250050946

[14] Yokoyama, I. (2013) Myocardial insulin resistance is not always pararell skeletal muscle and whole body insulin resistance: A mini review-Myocardial insulin resistance. Journal of Biomedical Science and Engineering, 6, 3135. doi:10.4236/jbise.2013.61005

[15] Nuutila, P., Koivisto, V.A., Knuuti, J., et al. (1992) Glucose-free fatty acid cycle operates in human heart and skeletal muscle in vivo. The Journal of Clinical Investigation, 89, 1767-1774. doi:10.1172/JCI115780

[16] Patlak, C.S., Blasberg, R.G. and Fenstermacher, J.D. (1983) Graphical evaluation of blood-to-brain transfer constants from multiple-time uptake data. Journal of Cerebral Blood Flow \& Metabolism, 3, 1-7. doi:10.1038/jcbfm.1983.1

[17] Gambhir, S.S., Schwaiger, M., Huang, S.C., et al. (1989) Simple noninvasive quantification method for measuring myocardial glucose utilization in humans employing positron emission tomography and fluorine-18 deoxyglucose. Journal of Nuclear Medicine, 30, 359-366.

[18] Ohtake, T., Kosaka, N., Watanabe, T., et al. (1991) Noninvasive method to obtain input function for measuring tissue glucose utilization of thoracic and abdominal organs. Journal of Nuclear Medicine, 32, 1432-1438.

[19] Yokoyama, I., Yonekura, K., Moritan, T., et al. (2001) Toroglitazone can improve impaired femoral muscle glucose utilization in type II diabetics with or without hypertension. Journal of Nuclear Medicine, 42, 1005-1010.

[20] Yokoyama, I., Inoue, Y., Moritan, T., Ohtomo, K. and Nagai, R. (2005) Measurement of skeletal muscle glucose utilization by dynamic 18F-FDG PET without arterial blood sampling. Nuclear Medicine Communications, 26, 31-37. doi:10.1097/00006231-200501000-00006

[21] Yokoyama, I., Inoue, Y., Moritan, T., Ohtomo, K. and Nagai, R. (2003) Simple quantification of skeletal muscle glucose utilization by static $18 \mathrm{~F}-\mathrm{FDG}$ PET. Journal of Nuclear Medicine, 44, 1592-1598.

[22] Ehrenkaufer, R.E., Potocki, J.F. and Jewett, D.M. (1984) Simple synthesis of F-18-labeled 2-fluoro-2-deoxy-D-glucose: Concise communication. Journal of Nuclear Medicine, 25, 333-337.

[23] Kelley, D.E., Williams, K.V., Price, J.C. and Goodpaster,
B. (1999) Determination of the lumped constant for $\left[{ }^{18} \mathrm{~F}\right]$ fluorodeoxyglucose in human skeletal muscle. Journal of Nuclear Medicine, 40, 1798-1804.

[24] Peltoniemi, P., Lönnroth, P., Laine, H., et al. (2000) Lumped constant for $\left[{ }^{18} \mathrm{~F}\right]$ fluorodeoxyglucose in skeletal muscles of obese and nonobese humans. American Journal of Physiology. Endocrinology Metabolism, 279, E1122-E1130.

[25] Yokoyama, I., Ohtake, T., Momomura, S., et al. (1998) Hyperglycemia rather than insulin resistance is related to coronary flow reserve in patients with non-insulin dependent diabetes mellitus. Diabetes, 47, 119-124. doi:10.2337/diabetes.47.1.119

[26] Lackner, R., Challiss, R.A., West, D. and Newsholme, E.A. (1984) A problem in the radiochemical assay of glucose6-phosphatase in muscle. The Biochemical Journal, 218, 649-651.

[27] Yokoyama, I., Ohtake, T., Momomura, S., Nishikawa, J., Sasaki, Y. and Omata, M. (1996) Reduced coronary flow reserve in hypercholesterolemic patients without overt coronary stenosis. Circulation, 94, 3232-3238. doi:10.1161/01.CIR.94.12.3232

[28] Yokoyama, I., Momomura, S., Ohtake, T., et al. (1998) Altered myocardial vasodilatation in patients with hyperiglyceridemia in anatomically normal coronary arteries. Arteriosclerosis Thrombosis and Vascular Biology, 18, 294-299. doi:10.1161/01.ATV.18.2.294

[29] Yokoyama, I., Ohtake, T., Momomura, S., et al. (1997) Reduced myocardial flow reserve in patients with noninsulin dependent diabetes mellitus. Journal of the American College of Cardiology, 30, 1472-1477. doi:10.1016/S0735-1097(97)00327-6

[30] Yokoyama, I., Momomura, S., Ohtake, T., et al. (1999) Improvement of myocardial vasodilatation in hypelipidemics due to diffuse coronary arterosclerosis after lipid lowering therapy. Circulation, 100, 117-122. doi:10.1161/01.CIR.100.2.117

[31] Yokoyama, I., Inoue, Y., Moritan, T., Ohtomo, K. and Nagai, R. (2004) Impaired myocardial vasodilatation during hyperaemic stress is improved by simvastatin but not by pravastatin in patients with hypercholesterolaemia. European Heart Journal, 25, 671-679. doi:10.1016/j.ehj.2004.02.017

[32] Van der Weerdt, A.P., Klein, L.J., Visser, C.A., Visser, F.C. and Lammertsma, A.A. (2002) Use of arterialised venous instead of arterial blood for measurement of myocardial glucose metabolism during euglycaemic-hyperinsulinaemic clamping. European Journal of Nuclear Medicine and Molecular Imaging, 29, 663-669. doi:10.1007/s00259-002-0772-y

[33] Iida, H., Miura, S., Shoji, Y., et al. (1998) Non-invasive quantitation of CBF using oxygen-15-water and a dualPET system. Journal of Nuclear Medicine, 39, 17891798.

[34] Ito, H., Kinoshita, T., Tamura, Y., Yokoyama, I. and Iida, H. (1999) Effect of intravenous dipyridamole on cerebral blood flow in humans: A PET study. Stroke, 30, 16161620. doi:10.1161/01.STR.30.8.1616

[35] Ito, H., Yokoyama, I., Iida, H., et al. (2000) Regional differences in cerebral vascular response to $\mathrm{PaCO}_{2}$ 
changes in humans measured by PET. Journal of Cerebral Blood Flow \& Metabolism, 20, 1264-1270.

[36] Yokoyama, I., Inoue, Y., Kinoshita, T., Itoh, H., Kanno, I. and Iida, H. (2008) Heart and brain circulation and $\mathrm{CO}_{2}$ in healthy men. Acta Physiologica, 193, 303-308. doi:10.1111/j.1748-1716.2008.01846.x

\section{ABBREVIATIONS}

SMGU: Skeletal muscle glucose utilization;

PET: Positron emission tomography;

${ }^{18}$ F-FDG: ${ }^{18}$ Fluorine-fluoro-2-deoxy-D-glucose;

CAD: Coronary artery disease;

WBGR: Whole body glucose disposal rate;

MGU: Myocardial glucose utilization;
ROIs: Regions of interest;

FWHM: Full width at half maximum;

LC: Lumped constant;

$\mathrm{Gl}_{\mathrm{p}}$ : Mean of the venous plasma glucose level during imaging;

RC: Recovery coefficients. 